\title{
A Rotational Sector-Based, Receiver-Oriented Mechanism for Location Tracking and Medium Access Control in Ad Hoc Networks Using Directional Antenna
}

\author{
Tetsuro Ueda ${ }^{1}$, Shinsuke Tanaka ${ }^{1}$, Dola $\mathrm{Saha}^{2}$, Siuli Roy ${ }^{2}$, \\ and Somprakash Bandyopadhyay ${ }^{2}$ \\ ATR Adaptive Communication Research Laboratories \\ 2-2-2 Hikaridai, Seika-cho Soraku-gun, Kyoto 619-0288 Japan \\ \{teueda, shinsuke\} atr.co.jp \\ ${ }^{2}$ Indian Institute of Management Calcutta \\ Diamond Harbour Road, Joka Calcutta 700104 India \\ \{dola, siuli, somprakash\}@iimcal.ac.in
}

\begin{abstract}
The use of directional antenna in wireless ad hoc networks potentially increases simultaneous communication by directing the transmitting and receiving beams towards the receiver and transmitter node as compared to omni-directional antenna, where nodes in the vicinity of a communication are kept silent. However, in order to implement effective directional MAC protocol using directional antenna, a node should know how to set its transmission direction to transmit a packet to its neighbors and to avoid transmission in other directions where data communications are already in progress. So, it becomes imperative to have a mechanism at each node to track the locations of its neighbors and to know the communication status of neighboring nodes. In this paper, we propose a receiver-centric approach for location tracking and MAC protocol. The performance evaluation on QualNet network simulator indicates that our protocol is highly efficient with increasing number of communications and increasing data rate.
\end{abstract}

\section{Introduction}

The recent progress in wireless communication and personal computing leads to the research of ad hoc wireless networks, which are envisioned as rapidly deployable, infrastructure-less networks with each node acting as a mobile router, equipped with a wireless transceiver. Usually, in ad hoc networks, all nodes are equipped with omnidirectional antenna. However, ad hoc networks with omni-directional antenna uses RTS/CTS based floor reservation scheme that wastes a large portion of the network capacity by reserving the wireless media over a large area. Consequently, lot of nodes in the neighborhood of transmitter and receiver has to sit idle, waiting for the data communication between transmitter and receiver to finish. To alleviate this problem, researchers have proposed to use directional (fixed or adaptive) antennas that direct the transmitting and receiving beams toward the receiver and transmitter node only. This would largely reduce radio interference, thereby improving the utilization of wireless medium and consequently the network throughput [1-11]. 
In order to fully exploit the capability of directional antenna, it is necessary for each node to know the information of the neighboring nodes (such as node-ID, direction, link quality, etc.) beforehand. Thus, in order to implement effective MAC and routing protocol in this context, a node should know how to set its transmission direction to transmit a packet to its neighbors. So, it becomes imperative to have a mechanism at each node to track the locations of its neighbors. However, this location tracking mechanism in the context of wireless ad hoc networks with directional antenna is a serious problem, since it incurs a lot of control overhead. In this paper, we are proposing a receiver-centric approach for location tracking and MAC protocol. In order to track the location of its neighbor, each node $\mathrm{n}$ periodically collects its neighborhood information and forms an Angle- Signal Table (AST). Based on AST, a node $\mathrm{n}$ knows the direction of node $\mathrm{m}$ and controls the medium access during transmission-reception. The performance evaluation on QualNet network simulator [12] indicates that our protocol is highly efficient with increasing number of communications and with increase in data rate. The one-hop MAC throughput of our protocol is 1.8 times as compared to that of IEEE 802.11. Moreover, average number of data packet retransmission due to loss of data packets is substantially less (less than $5 \%$ of that with IEEE 802.11).

\section{Related Work}

In spite of the advantages of directional antennas, work on developing efficient MAC protocol using directional antennas in the context of ad hoc networks is limited because of the inherent difficulty to cope up with mobility and de-centralized control in ad hoc networks. Some researchers in the past have tried to address this challenge in several ways $[1,2]$. In recent years, several MAC protocols that rely on RTS-CTS type handshaking as in IEEE 802.11 have been suggested with directional antennas [3-11]. In [3], a set of D-MAC (Directional MAC) schemes has been proposed where combination of directional/ omni-directional RTS / CTS are used to block nodes from transmitting in directions that would interfere with existing data transmission while allowing them to transmit on other directions. In [4], a MAC protocol to achieve multihop efficiency has been proposed with multihop-RTS-singlehop-CTS using directional antenna. In this mechanism, using larger range of directional beam, a destination is reachable in less number of hops as compared to that using omni directional antenna. In both the schemes [3-4], the mobile nodes are assumed to know the physical locations of themselves and their neighbors using GPS. In [5], the proposed MAC protocol need not know the location information; the source and destination nodes identify each other's direction during omni-directional RTS-CTS exchange in an on-demand basis. In [6], Ramanathan studied the performance of ad hoc networks using beamforming antennas with changing antenna patterns and beam control, channel access mechanisms, link power control and neighbor discovery. The authors assume prior knowledge of location information. In [7], concept of Directional NAV (the network allocation vector) has been used, one for each sector, allowing immediate transmission of control packets on those sectors which are clear instead of having to defer the transmission until it is safe to transmit on all sectors at the same time. In [8] also, Takai et al. proposed a directional NAV with a direction and a width, which is set depending on the signal strength in that direction. 
Developing a suitable MAC protocol in ad hoc network to exploit the advantages of directional antenna for overall performance improvement requires proper location tracking and neighborhood knowledge. In [5], the source and destination nodes identify each other's direction during omni-directional RTS-CTS exchange. However, in this mechanism, a node is not aware of its complete neighborhood information. In [3-4], the use of GPS is proposed to track the location of each node but the exact mechanism of information exchange and the consequent overhead has not been discussed. In our earlier work, we have developed a MAC protocol [9], where each node keeps certain neighborhood information dynamically through the maintenance of an Angle-SINR Table. In this method, in order to form AST, each node periodically sends a directional beacon in the form of a directional broadcast, sequentially in all direction at 30 degree interval, covering the entire 360 degree space. The nodes, which receive these signals at different angles, determine the best received signal strength and transmit the information back to the source node as data packet with RTS/CTS handshake. However, the overhead due to control packets is very high in this method [9] of location tracking.

In this paper, we will illustrate a receiver-oriented location tracking mechanism to reduce the control overhead, which is described in Section 4 and a simple MAC protocol for efficient medium utilization, which is depicted in Section 5. We have done extensive performance evaluation using QualNet to demonstrate its effectiveness and is illustrated in Section 6.

\section{System Description}

\subsection{Antenna Model}

There are basically two types of smart antennas used in the context of wireless networks: switched-beam or fixed beam antennas and steerable adaptive array antennas [12,13,14]. A switched-beam antenna generates multiple pre-defined fixed non-overlapping directional beam-patterns and applies one at a time when receiving a signal. Signals will be sensed in all sectors and the antenna is capable of recognizing the sector with the maximum gain. When receiving, exactly one sector, which usually is the one chosen by the sensing process, will collect the signals.

In a steerable adaptive array antenna, the beam structure adapts to Radio Frequency (RF) signal environment and directs beams towards the signal of interest to maximize the antenna gain, simultaneously depressing the antenna pattern (by setting nulls) in the direction of the interferers [14]. In adaptive array antennas, an algorithm is needed to control the output, i.e. to maximize the Signal to Interference and Noise Ratio (SINR).

We have developed a wireless ad hoc network testbed using smart antenna [15] where each user terminal uses a small, low-cost smart antenna, known as ESPAR (Electronically Steerable Passive Array Radiator) antenna [16,17]. The adaptive array antennas are normally digital beamforming antennas. On the other hand, ESPAR antenna relies on RF beamforming, which drastically reduces the circuit complexity. Since ESPAR antenna requires only one receiver chain, it is possible to provide drastic improvement in both dc power dissipation and fabrication costs, by eliminating the need for frequency converters and analog-digital converters by the 
number of array branches [16]. The features of ESPAR are: controlling beam direction, multiple beams (with same frequency) formation, steerable beam (360 degree sweeping) and controlling null steering. For receiver application, the null should be steered in the direction from which an interfering signal is coming.

Developing suitable MAC protocols with adaptive antenna in ad hoc networks is a challenging task. That is why, most of the works in the context of ad hoc networks assume to use simpler switched beam antenna. In this work also, we are using smart ESPAR antenna as a switched beam antenna. ESPAR antenna can also be used as a generalized switched beam antenna or quasi-switched beam antenna, by selecting the value of reactance for one specific directional beam among multiple directional beam patterns, without using multiple receiver chains (frequency converters and analogdigital converters). By including some mechanism to detect direction of arrival (DoA) for the signal received from the user (as will be illustrated shortly), continuous tracking can be achieved and it can be viewed as a generalization of the switched beam concept [14]. In this case also, the received power is maximized. The advantage of using ESPAR antenna as generalized switched beam antenna is that, with only one receiver chain, continuous tracking is possible and we can have variable number of beam-pattern. Since ESPAR antenna would be a low-cost, low-power, small-sized antenna, it would help to reduce the power consumption of the user terminals in WACNet and would be able to deliver all the advantages of switched beam antenna.

\subsection{A Few Assumptions and the Rationales}

- When the antenna of a node operating in omni-directional mode, it is capable of transmitting and receiving signal from all direction with a gain, say, $\mathrm{G}^{\text {omni }}$. While idle, a node operates in omni-directional receive mode.

- When the antenna of a node operating in directional mode, a node can points its beam (main lobe) towards a specified direction with beam width $w$ and with a gain, say $G^{\text {dir }}\left(G^{\text {dir }}>>G^{\text {omni }}\right)$. Beam width is around 60 degree in our simulation.

- Consequently, for a given amount of input power, the transmission range $\mathrm{R}^{\mathrm{dir}}$ with directional antenna will be much larger than that with corresponding omnidirectional antenna $\left(\mathrm{R}^{\mathrm{omni}}\right)$.

- We define neighbors of a node $\mathrm{n}$ as a set of nodes within the omni-directional transmission range of $\mathrm{n}$. From the perspective of directional data communication, it implies that a neighbor, say, $\mathrm{m}$ of a node, say, $\mathrm{n}$ is always a strong neighbor. Hence, the received signal strength at $m$ from $n$ is always high to ensure proper capture even in presence of other interference. Thus, the chance of $\mathrm{m}$ getting disconnected or weakly connected during a data packet transfer from $n$ due to an outward mobility of either $\mathrm{m}$ or $\mathrm{n}$ is far less.

- This will alleviate the problem of hidden terminal in this context as indicated in [4]. Let us consider Fig. 1 where node $n$ is communicating with node $m$ with directional beam. Node $\mathrm{p}$ now wants to communicate with node $\mathrm{q}$. If node $\mathrm{p}$ is within the neighborhood of $n$, this communication will not be initiated, since $p$ is not allowed to form directional beam towards $n$ and/or $m$. However, if node $p$ is outside the neighborhood of $\mathrm{n}$, node $\mathrm{p}$ forms a directional beam towards node $\mathrm{q}$ and starts communication. This may interfere with node m's reception. However, since the distance $\mathrm{p}$ and $\mathrm{m}$ is larger than $\mathrm{n}$ and $\mathrm{m}$ by at least $\mathrm{R}^{\text {omni }}$ (the omni- 
directional range), the received signal at $\mathrm{m}$ from $\mathrm{n}$ will predominate and chance of data packets being lost due to this interference will be insignificant. This is reflected in our simulation results, which shows very low packet retransmission under different conditions.

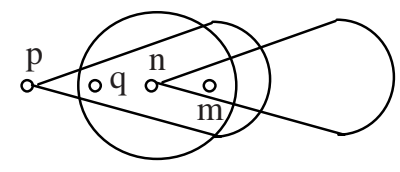

Fig. 1. The capture of receiver $m$ by transmitter $n$ is strong enough to tolerate interference from another transmitter $\mathrm{p}$

However, as a consequence of this assumption, we are sacrificing multihop efficiency which could have been achieved using directional antenna, since using larger range of directional beam, a destination is reachable in fewer number of hops as compared to that using omni directional antenna. However, what we are gaining is SDMA efficiency, as will be demonstrated in the performance evaluation.

\section{Location Tracking Mechanism}

In this study, each node waits in omni-directional-receive-mode while idle. Whenever it senses some signal above a threshold, it enters into rotational-sector-receive-mode. In rotational-sector-receive mode, node $\mathrm{n}$ rotates its directional antenna sequentially in all direction at 30 degree interval, covering the entire 360 degree space in the form of the sequential directional receiving in each direction and senses the received signal at each direction. After one full rotation, it decides the best possible direction of receiving the signal with maximum received signal strength. Then it sets its beam to that direction and receives the signal.

However, in order to enable the receiver decoding the received signal, each control packet is transmitted with a preceding tone with a duration such that the time to rotate a receiver's rotational receive beam through 360 degree is less than the duration of the tone. The purpose of this transmitted tone before any control packet is to enable the receiver to track the best possible direction of receiving the signal. Once it sets its beam to that direction, the purpose of tone signal is over and subsequently the control packet is transmitted.

In this proposed framework, we have used three types of control packets: beacon or "hello" packet used to track the location of neighboring nodes), RTS (Request to send) and CTS (clear to send) for medium access control. Beacon is a periodic signal, transmitted from each node at a pre-defined interval. At each periodic interval, each node, say, m, sends an omni-directional beacon to its neighbors, if the medium is free. As indicated earlier, each beacon is transmitted with a preceding tone signal that helps the receivers to detect the best possible direction of receiving the beacon. Then each receiver sets its beam to that direction and receives and decodes the beacon. Thus, the node $\mathrm{n}$ which is, say, a neighbor of $\mathrm{m}$ forms the Angle-Signal information for node $\mathrm{m}$, and similarly, for other neighbors. An entry in AST of node $\mathrm{n}$ for its neighbor $\mathrm{m}$ is SIGNAL $_{n, m}^{\alpha}(\mathrm{t})$, which is the maximum strength of received signal at node $\mathrm{n}$ from 
node $\mathrm{m}$ at an angle $\alpha$ with respect to $\mathrm{n}$ and as perceived by $\mathrm{n}$ at any point of time $\mathrm{t}$. Based on AST, a node $\mathrm{n}$ knows the direction of node $\mathrm{m}$ and controls the medium access during transmission-reception.

Since RTS is a broadcast packet and contains source address, nodes can decode that RTS also to form the Angle-Signal Table. So, we have used RTS as beacon. If an RTS is sent, beacon timer is reset. The use of RTS as beacon is advantageous at high traffic where overhead due to beacon is minimized. This is because, the transmitting nodes don't have to send an additional beacon to inform its neighbors of its presence.

\section{Directional Medium Access Control Protocol}

In IEEE 802.11 MAC protocol standard, RTS-CTS-DATA-ACK exchange mechanism is used to ensure reliable data communication. In our scheme, initially, when node $\mathrm{n}$ wants to communicate with $\mathrm{m}$, it senses the medium and if it is free, sends omni-directional RTS. The back-off mechanism is same as in IEEE 802.11. The purpose of RTS is to inform all the neighbors of $\mathrm{n}$, including $\mathrm{m}$, that a communication from $\mathrm{n}$ to $\mathrm{m}$ has been requested. It also specifies the approximate duration of communication. All the neighboring nodes of $\mathrm{n}$ keep track of this request from node $\mathrm{n}$, whose direction is known to the each of them from the received RTS signal. The mechanism for receiving RTS is same as that for beacon.

The target node $\mathrm{m}$ sends omni-directional CTS to grant the request and to inform the neighbors of $\mathrm{m}$ that $\mathrm{m}$ is receiving data from $\mathrm{n}$. It also specifies the approx. duration of communication. All the neighboring nodes of $\mathrm{m}$ keep track of the receiving node $\mathrm{m}$, whose direction is known to the each of them from the received CTS signal. Once again, the mechanism for receiving CTS is same as that for beacon. It is to be noted that the objective of RTS/CTS here is not to inhibit the neighbors of $n$ from transmitting or receiving (as is the case with omni-directional antenna) but to inform the neighbors of $\mathrm{n}$ that $\mathrm{m}$ is receiving data from $\mathrm{n}$.

After transmission of omni-directional CTS, the receiving node waits in directional receive mode until Data is transmitted or timeouts and returns to omni-directional receive mode. Also, once the CTS is received, the transmitter transmits Data directionally and waits for Acknowledgement directionally until Acknowledgement is received or timeouts and returns to omni-directional receive mode. The receiver on receiving Data, transmits Acknowledgement directionally and returns to omnidirectional receive mode. The directional reception mode ensures proper reception of signal from the required direction and minimization of interference from other direction.

Other nodes in the neighborhood of $\mathrm{n}$ and $\mathrm{m}$, who overheard the RTS/CTS exchange, set their Directional Network Allocation Vector (DNAV) in the direction which they detected as the direction of arrival of the RTS or CTS respectively. Now, if they have a packet to send to a node, whose direction as known from AST, is not in the direction of blocked DNAV, then they can issue both RTS and CTS omnidirectionally without disturbing the communication between $n$ and $m$. Omnidirectional RTS/CTS will not create interference at the receiver end of the on-going communication because receiver directs its receiving beam pattern towards the transmitter. If the direction of receiving node is blocked by DNAV and RTS is issued, it is most probable that CTS will not be issued or their may be RTS collision. As a 
result, the node will increase its contention window and enter into backoff. This may happen repeatedly and as a result, the node will get less chance to transmit. So, we do not allow transmission of RTS in this case. Here, the node waits for DNAV time and then tries to start communication, which is similar to waiting for NAV as explained in standard IEEE 802.11.

\section{Performance Evaluation}

\subsection{Simulation Environment}

The simulations are conducted using QualNet 3.1 [12]. We have simulated ESPAR antenna in the form of a quasi-switched beam antenna, which is steered discretely at an angle of 30 degree, covering a span of 360 degree. We have simulated our MAC protocol with (i) Simulated ESPAR Antenna Pattern (ESPAR), (ii) QualNet's default directional antenna pattern (DEFAULT) and (iii) an Ideal directional antenna pattern without sidelobes (IDEAL) as described in Section 3.1. We have done the necessary changes in QualNet simulator to implement Directional Virtual Carrier Sensing in MAC Layer and directional transmission in Physical Layer of QualNet simulator.

ESPAR antenna requires 16 microseconds to measure the level of received signal in each sector at $2 \mathrm{Mbps}$ data rate and 10 nanoseconds to change the beam pattern from one sector to another. So, to rotate in 12 patterns and detect the gain in each antenna pattern, ESPAR antenna requires nearly $[(12 \times(16+0.01))$ microseconds $]=$ 192.12 microseconds. Hence, in our simulation, we have chosen the duration of preceding tone in control packets to be 200 microseconds.

We have used simple one-hop randomly chosen communication in order to avoid the effects of routing protocols to clearly illustrate the difference between 802.11 and our proposed MAC. Also, we have used static routes to stop all the control packets generated by any routing protocol, whether it is proactive or reactive. In our simulation, we studied the performance of the proposed MAC protocol in comparison with the existing omnidirectional 802.11 MAC protocol by varying the data rate and number of simultaneous communications. In studying our MAC protocol, we have used different antenna patterns as described above to ensure the robustness of our proposed MAC protocol. In doing this, we have used ESPAR antenna as one of the antenna patterns, to evaluate the performance of the ESPAR antenna as well. The set of parameters used are listed in Table 1.

Table 1. Parameters used in Simulation

\begin{tabular}{|l|l|}
\hline Parameters & Value \\
\hline Area & $1000 \times 1000 \mathrm{~m}$ \\
\hline Number of nodes & 40 \\
\hline Transmission Power & $15 \mathrm{dBm}$ \\
\hline Receiving Threshold & $-81.0 \mathrm{dBm}$ \\
\hline Sensing Threshold & $-91.0 \mathrm{dBm}$ \\
\hline Data Rate & $2 \mathrm{Mbps}$ \\
\hline Packet Size & 512 bytes \\
\hline Duration of Preceding Tone & 200 microseconds \\
\hline CBR Packet Arrival Interval & 2 ms to $50 \mathrm{~ms}$ \\
\hline Number of simultaneous communication & 2 to 16 \\
\hline Simulation Time & 5 minutes \\
\hline
\end{tabular}




\subsection{Results and Discussions}

We have used the existing IEEE 802.11 MAC, which we caption as "802.11", as a benchmark to compare and evaluate the performance of our proposed MAC protocol with ESPAR antenna, QualNet's default antenna and an ideal antenna, which we caption as "ESPAR", "DEFAULT", and "IDEAL" respectively. Our evaluation is based on two criteria: Average Throughput, and One Hop Average End-to-End Delay. The results are shown in Fig. 2 and 3 respectively. Each result reported is an average of ten executions with different seeds. So, to complete our results, we had to simulate over 400 scenarios, each of which was executed in the simulator for 5 minutes to get an overall average result.

MAC Performance depends much on antenna pattern also. So, we have simulated for 3 different types of antenna patterns. In showing the results with three beam patterns, we had two notions in our mind: (a) E-MAC performs nicely with directional antenna and maximum gain that can be obtained from E-MAC is nearly thrice than that of IEEE 802.11, if beam-width of directional antenna is 45 degree and sectors are 45 degree each, and (b) In context of an ideal antenna pattern with 45 degree beamwidth, which gives maximum gain, performance of ESPAR antenna with 60 degree beamwidth is quite reasonable and it even performs better than a standard antenna pattern with 45 degree beamwidth provided by QualNet Simulator.

In Fig. 2, it is observed that with increasing data rate, average throughput of our proposed MAC protocol with any directional antenna pattern is much better than that of IEEE 802.11 and one hop average end-to-end delay of E-MAC is nearly half of that obtained with IEEE 802.11 protocol. In omnidirectional 802.11, nodes have to sit idle in the omni-directional range of both transmitter and receiver to facilitate the ongoing communication. But, with the use of directional antenna, and the implementation of Directional Virtual Carrier Sensing, E-MAC creates an environment of lower contention which "802.11" cannot create with an omnidirectional antenna. Hence, with increasing data rate, average throughput increases sharply in E-MAC as evident from Fig. 2. Also, we can see that due to SDMA efficiency in E-MAC, less contension in E-MAC leads to low one hop average end-to-end delay. With ESPAR antenna, this delay is nearly half that of IEEE 802.11 protocol.

In Fig. 3, it is observed that with increasing number of simultaneous communication, average throughput decreases in both E-MAC and 802.11, but EMAC shows significant gain in Average Throughput. This is because E-MAC does not inhibit neighboring nodes to transmit, but just informs neighbors of the ongoing communication and its direction, so that they can start communication in other directions. But 802.11 with omnidirectional antenna, keeps all neighboring nodes silent by issuing RTS/CTS. Also, with increasing number of simultaneous communication, one hop average end-to-end delay increases in both IEEE 802.11 and E-MAC, but the increase is much prominent in "802.11" than in E-MAC, irrespective of the directional antenna pattern used. With increasing number of simultaneous communication, interference to each communication increases due to interference of added number of simultaneous communication. But, E-MAC not only informs other nodes in its vicinity of the on-going communication, but also transmits and receives directionally, which minimizes interference from other directions also. Thus E-MAC exploits SDMA efficiency for which more number of simultaneous communication is possible, which leads to lesser queuing delay and lesser one hop average end-to-end delay as observed in Fig. 3. 

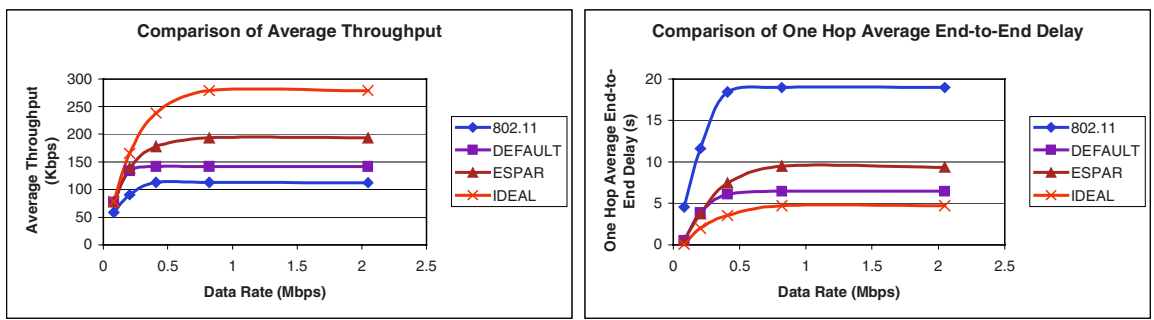

Fig. 2. Performance Evaluation of the proposed MAC protocol with directional antenna with increasing data rate
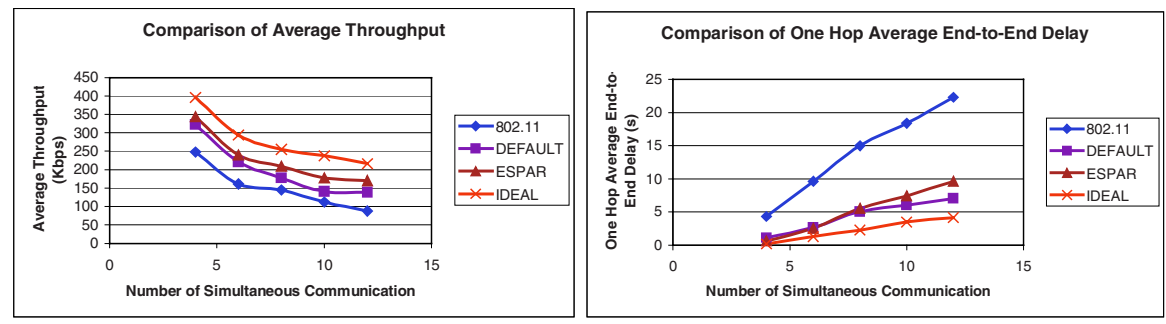

Fig. 3. Performance Evaluation of the proposed MAC protocol with directional antenna with increasing number of simultaneous communication

\section{Conclusion}

Use of directional antenna in ad hoc wireless network can drastically improve system performance, if proper MAC protocol can be designed. With directional setting of Virtual Carrier Sensing, medium can be utilized to its maximum with directional antenna. Even with the overhead of location tracking, gain obtained in MAC is really significant. Presently, we are working on efficient controlling of transmission power to improve the proposed MAC performance.

\section{References}

1. J. Zander, "Slotted ALOHA multihop packet radio networks with directional antennas", Electronic Letters, vol.26, no.25, 1990

2. T.S. Yum and K.W. Hung, "Design algorithms for multihop packet radio networks with multiple directional antennas stations," IEEE Transactions on communications, vol. 40, no. 11, pp. 1716-1724, 1992.

3. Y.B. Ko, V. Shankarkumar and N. H. Vaidya, "Medium access control protocols using directional antennas in ad hoc networks," Proc. of the INFOCOM 2000, March 2000.

4. Romit Roy Choudhury , Xue Yang , Nitin H. Vaidya , Ram Ramanathan, "Using directional antennas for medium access control in ad hoc networks" Proc. of the eighth annual international conference on Mobile computing and networking September 2002 
5. Nasipuri, S. Ye, J. You and R.E. Hiromoto, "A MAC Protocol for Mobile Ad Hoc Networks Using Directional Antennas", Proc of the IEEE WCNC 2000.

6. R. Ramanathan, "On the Performance of Ad Hoc Networks with Beamforming Antennas", ACM MobiHoc, October 2001.

7. Kou Kobayashi and Masao Nakagawa, "Spatially divided channel scheme using sectored antennas for CSMA/CA - directional CSMA/CA", Proc.of PIMRC'2000, 2000.

8. M. Takai, J. Martin, R. Bagrodia and A. Ren, "Directional Virtual Carrier Sensing for Directional Antennas in Mobile Ad Hoc Networks", ACM MobiHoc, June 2002.

9. S. Bandyopadhyay, K. Hasuike, S. Horisawa, S. Tawara, "An Adaptive MAC Protocol for Wireless Ad Hoc Community Network (WACNet) Using Electronically Steerable Passive Array Radiator Antenna", Proc of the GLOBECOM 2001, November 25-29, 2001, San Antonio, Texas, USA

10. S. Bandyopadhyay, K. Hasuike, S. Horisawa, S. Tawara, "An Adaptive MAC and Directional Routing Protocol for Ad Hoc Wireless Network Using Directional ESPAR Antenna" Proc of the ACM Symposium on Mobile Ad Hoc Networking \& Computing 2001 (MOBIHOC 2001), Long Beach, California, USA, 4-5 October 2001

11. Asis Nasipuri, Kai Li, and Uma Reddy Sappidi, "Power Consumption and throughput in Mobile Ad Hoc Networks using Directional Antennas" in Proceedings of the IEEE International Conference on Computer Communication and Networks (ICCCN2002), October 14-16, 2002, Miami, Florida.

12. QualNet Simulator Version 3.1, www.scalable-networks.com

13. J.C.Liberti, T.S.Rappaport, "Smart Antennas for Wireless Communications: IS-95 and Third Generation CDMA Applications", Prentice-Hall, 1999.

14. Per H. Lehne and Magne Pettersen, An Overview of Smart Antenna Technology for Mobile Communications Systems, IEEE Communications Surveys, http://www.comsoc.org/pubs/surveys, Fourth Quarter 1999, vol. 2 no. 4.

15. T. Ueda, K. Masayama, S. Horisawa, M. Kosuga, K. Hasuike, "Evaluating the Performance of Wireless Ad Hoc Network Testbed With Smart Antenna", Fourth IEEE Conference on Mobile and Wireless Communication Networks (MWCN), September 2002

16. T. Ohira, "Adaptive array antenna beamforming architectures as viewed by a microwave circuit designer", 2000 Asia-Pasific Microwave Conf., Sydney, Dec. 2000.

17. K. Gyoda and T. Ohira, "Beam and Null Steering Capability of ESPAR Antennas, Proc of the IEEE AP-S International Symposium, July 2000. 